\title{
Diphtheria: down but not out yet!
}

Vidushi Mahajan', Isha Kapoor ${ }^{1}$; Department of ${ }^{1}$ Pediatrics, Government Medical College and Hospital, Sector 32 , Chandigarh, India

Introduction: We hereby report a case of severe tonsillopharyngeal diphtheria in an unimmunised Indian child which was complicated by renal failure, myocarditis, palatal palsy and polyneuropathy.

\section{Case History:}

Clinical presentation: An 11-year old unimmunized boy presented with gradually progressive dysphagia and noisy breathing for last 5 days. On examination, the child had bull neck, stridor, and bilateral grade II tonsillar hypertrophy with overlying grayish white membrane which bled to touch. (Fig.1) His Albert stain and throat swab culture for Corynebacterium diphtheriae was negative.

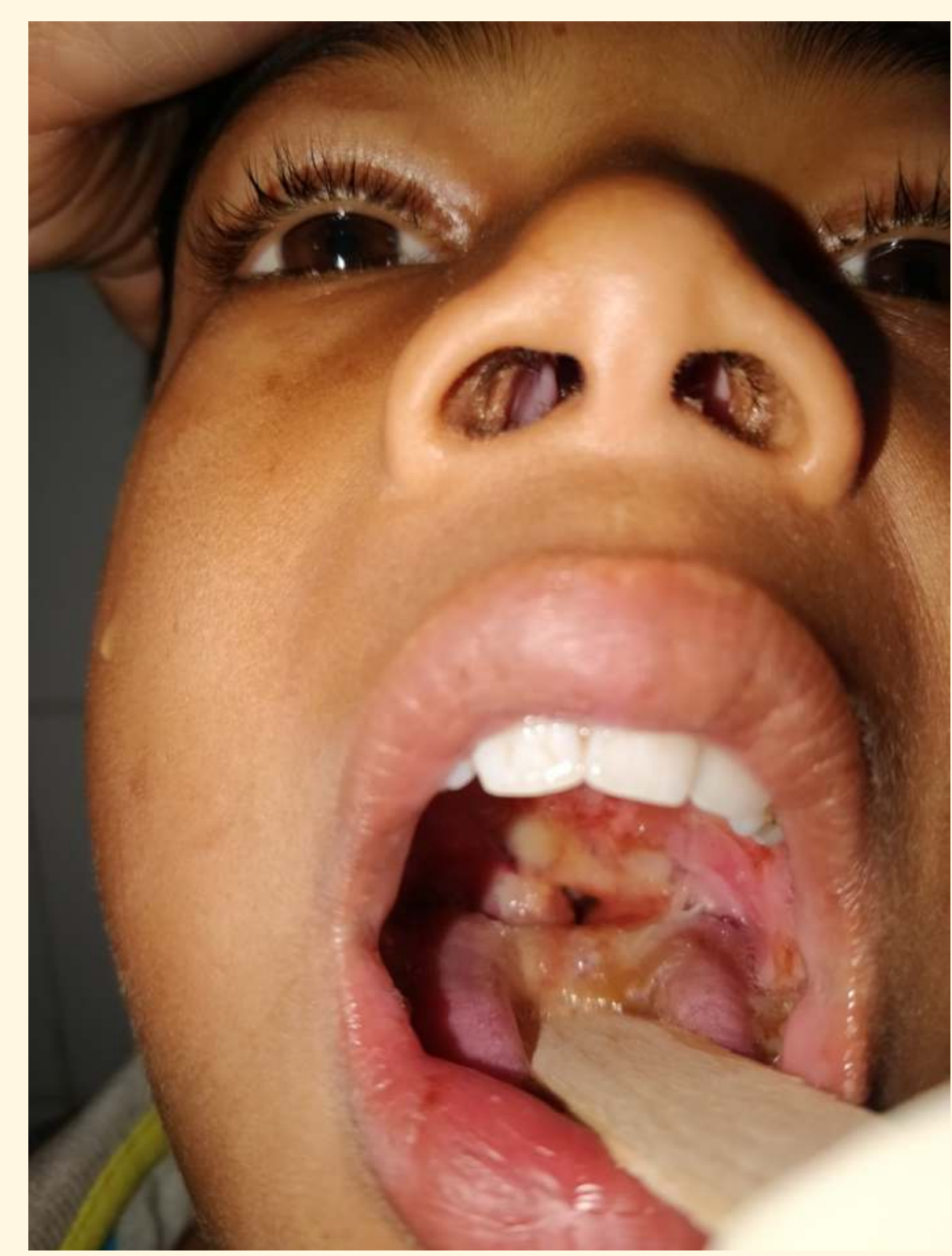

Fig.1 Pseudomembrane seen over tonsils and palate which bled on touch
Blood investigations revealed acute kidney injury (AKI) as seen in Fig.2. During the second week of hospitalisation, he developed hypotension, tachycardia and grade II systolic murmur consistent with myocarditis which was confirmed by creatine phosphokinase-MB levels, electrocardiogram and 2-D echocardiography. Two weeks post admission, he developed dysarthria, nasal twang of voice along with nasal regurgitation of liquids suggestive of palatal palsy. At 4 weeks, the child developed bilateral lateral gaze palsy along with paralysis of accommodation and diplopia. His clinical course is detailed in Fig. 3.

Management: Given his clinical presentation and unimmunised status, we considered the possibility of tonsillopharyngeal diphtheria. One lakh units of diphtheria anti-toxin was administered at admission and erythromycin was given for 14 days after which his bull neck, stridor amd pseudomembrane resolved. His worsening AKI required hemodialysis and hypotension necessitated the use of vasopressors. He was started on tube feeds in view of palatal palsy. He was closely monitored for onset of any diaphragmatic muscle weakness. His condition improved and at 7 weeks he was accepting orally with resolution of palatal palsy and diplopia and was discharged from the hospital.

Followup: The child was given catchup vaccination and is currently doing well with no neurological deficit.

Discussion: Diphtheria is an acute bacterial infection caused by toxin-producing strains of Corynebacterium spp.

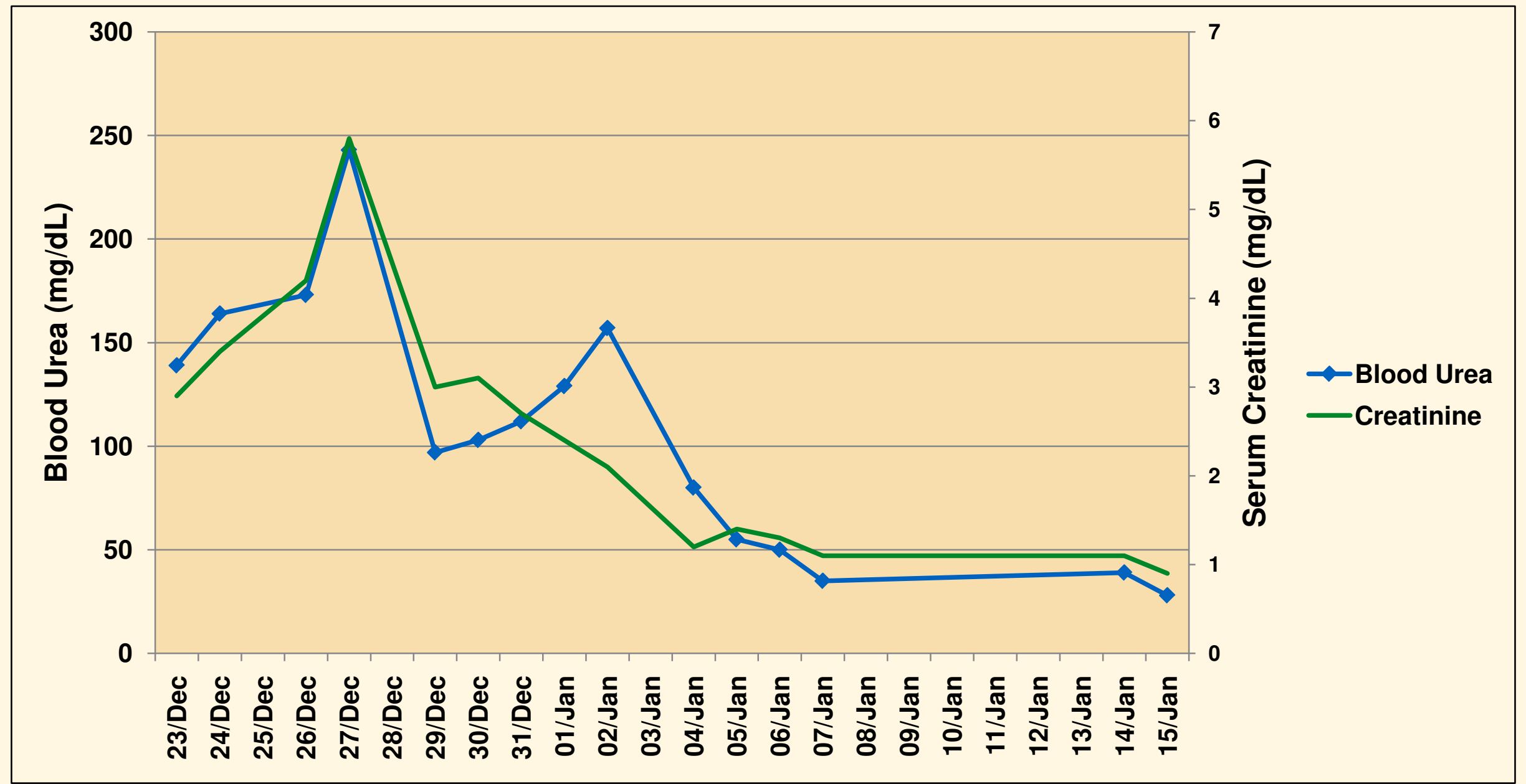

Fig.2 showing blood urea and serum creatinine trends in the child post hospitalisation

Diphtheria is characterised by formation of a pseudomembrane over the tonsils, pharunx, soft palate or uvula and multisystem involvement, often fatal. Other causes of membranous tonsillopharyngitis include infectious mononucleosis, streptococcal pharyngitis, infectious mononucleosis, and Vincent's angina. Routine immunisation outreach needs to be strengthened, as the disease burden would drastically reduce with adequate vaccine coverage

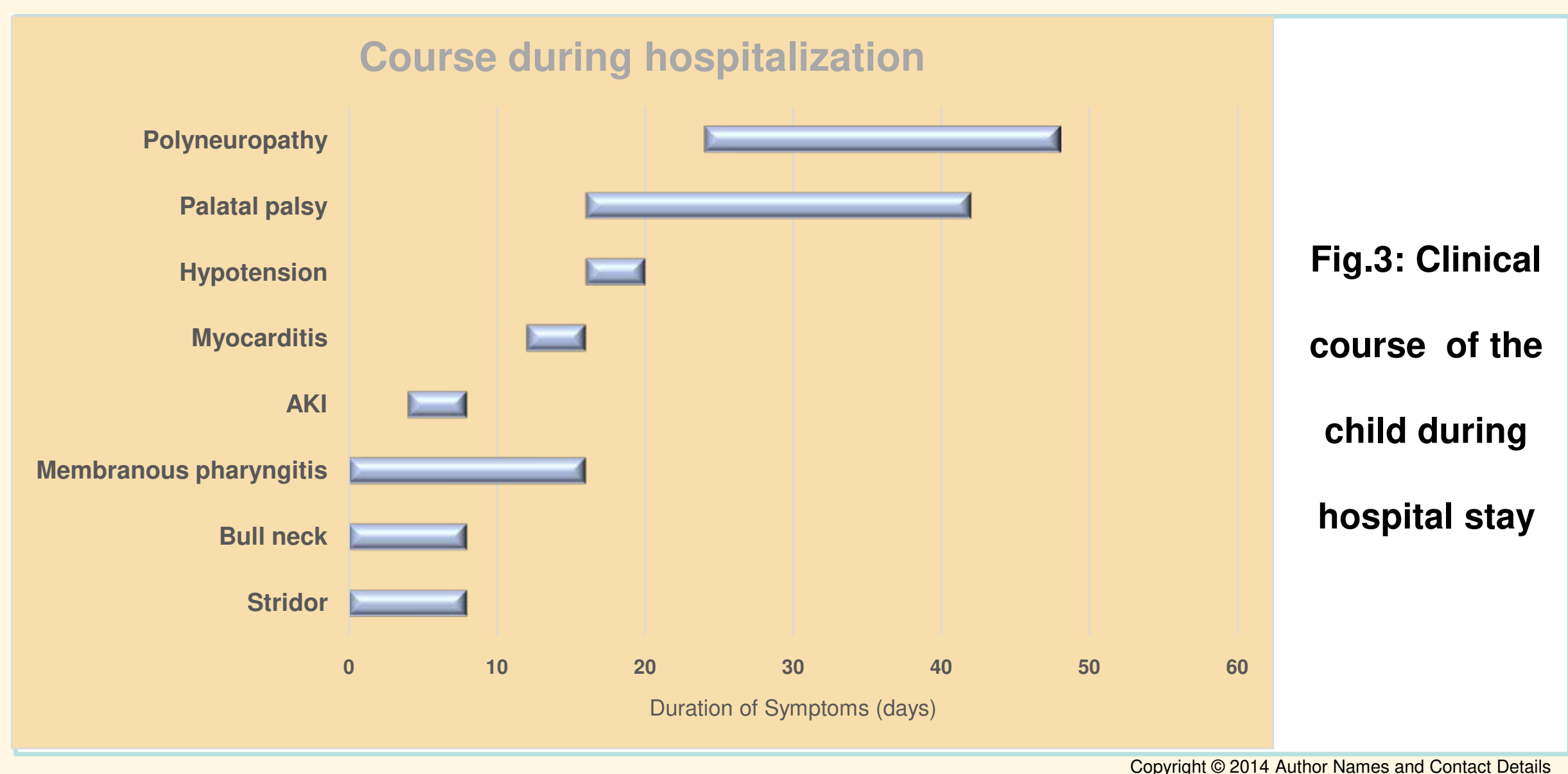

